\title{
Interrogating macromolecular complex assembly by systematically analyzing the composition of highly heterogeneous structural ensembles \\ Laurel Kinman ${ }^{1}$, Jingyu Sun ${ }^{2}$, Joaquin Ortega ${ }^{3}$, Joey Davis ${ }^{4}$ \\ ${ }^{1}$ Massachusetts Institute of Technology ${ }^{2}$ Center for Structural Biology, McGill University, ${ }^{3}$ Center for Structural Biology, McGill University, ${ }^{4}$ MIT \\ Ikinman@mit.edu
}

Cryo-EM represents a unique and powerful opportunity to structurally characterize biomolecules at the singleparticle level, and to draw biological insights from the heterogeneity observed within structural ensembles. Doing so, however, represents a significant computational challenge, and necessitates improved methods for studying extremely heterogeneous datasets. Here, we present an approach that combines our recently-published cryoDRGN method to reconstruct highly heterogeneous structural ensembles with a high-throughput compositional analysis that allows us to quantify the presence and absence of individual domains or whole proteins across hundreds-tothousands of cryo-EM density maps. This analysis produces a highly interpretable representation of the compositional heterogeneity present within a dataset. Using this representation, we can identify cooperative and mutually-exclusive occupancy relationships between various subunits, extract subsets of particles for traditional high-resolution refinement, and define pathways of structural change including complex assembly. We have applied this approach to understand the role of a universally-conserved methyltransferase in biogenesis of the 30S ribosomal subunit. By comparing the structural ensembles observed in the presence and absence of this factor, we have uncovered that this factor performs a novel proof-reading role in ribosome assembly. In sum, this work establishes a framework for systematically interrogating compositionally heterogeneous structural ensembles produced by tools such as cryoDRGN, and it highlights the value of this framework in illuminating underlying biological mechanisms. 